\title{
Clinical outcomes and predictors of response for adalimumab in patients with moderately to severely active ulcerative colitis: a KASID prospective multicenter cohort
} study

Seung Yong Shin ${ }^{1 *}$, Soo Jung Park ${ }^{2 *}$, Young Kim ${ }^{1}$, Jong Pil Im³ , Hyo Jong Kim ${ }^{4}$, Kang-Moon Lee ${ }^{5}$, Ji Won Kim, Sung-Ae Jung ${ }^{7}$, Jun Lee ${ }^{8}$, Sang-Bum Kang ${ }^{9}$, Sung Jae Shin ${ }^{10}$, Eun Sun Kim ${ }^{11}$, You Sun Kim ${ }^{12}$, Tae Oh Kim ${ }^{13}$, Hyun-Soo Kim ${ }^{14}$, Dong Il Park ${ }^{15}$, Hyung Kil Kim ${ }^{16}$, Eun Soo Kim ${ }^{17}$, Young-Ho Kim ${ }^{18}$, Do Hyun Kim ${ }^{19}$, Dennis Teng ${ }^{20}$, Jong-Hwa Kim ${ }^{21}$, Wonyong Kim ${ }^{21}$, Chang Hwan Choi ${ }^{1}$, on behalf of the IBD Research Group of the Korean Association for the Study of Intestinal Diseases

\begin{abstract}
${ }^{1}$ Department of Internal Medicine, Chung-Ang University College of Medicine, Seoul; ${ }^{2}$ Department of Internal Medicine and Institute of Gastroenterology, Yonsei University College of Medicine, Seoul, ${ }^{3}$ Department of Internal Medicine and Liver Research Institute, Seoul National University College of Medicine, Seoul; ${ }^{4}$ Department of Gastroenterology, Kyung Hee University Hospital, Seoul; ${ }^{5}$ Department of Gastroenterology, The Catholic University of Korea St. Vincent's Hospital, Suwon; ${ }^{6}$ Department of Gastroenterology, SMG-SNU Boramae Medical Center, Seoul; ${ }^{7}$ Department of Gastroenterology, Ewha Womans University College of Medicine, Seoul; ${ }^{8}$ Department of Internal Medicine, Chosun University College of Medicine, Gwangju; ${ }^{9}$ Department of Internal Medicine, Daejeon St. Mary's Hospital, The Catholic University of Korea College of Medicine, Daejeon; ${ }^{10}$ Department of Gastroenterology, Ajou University School of Medicine, Suwon, ${ }^{11}$ Department of Gastroenterology, Korea University Anam Hospital, Seoul; ${ }^{12}$ Department of Gastroenterology, Inje University Seoul Paik Hospital, Seoul; ${ }^{13}$ Department of Gastroenterology, Inje University Haeundae Paik Hospital, Busan; ${ }^{17}$ Department of Gastroenterology, Chonnam National University Hospital, Gwangju; ${ }^{15}$ Department of Internal Medicine, Kangbuk Samsung Hospital, Sungkyunkwan University School of Medicine, Seoul; ${ }^{16}$ Department of Gastroenterology, Inha University Hospital, Incheon; ${ }^{17}$ Department of Internal Medicine, School of Medicine, Kyungpook National University, Daegu; ${ }^{18}$ Department of Internal Medicine, Samsung Medical Center, Sungkyunkwan University School of Medicine, Seoul; ${ }^{19}$ AbbVie Ltd., Seoul, Korea; ${ }^{20}$ AbbVie Pte. Ltd., Singapore; ${ }^{21}$ Department of Microbiology, Chung-Ang University College of Medicine, Seoul, Korea
\end{abstract}

Background/Aims: This study assessed the efficacy and safety of adalimumab (ADA) and explored predictors of response in Korean patients with ulcerative colitis (UC). Methods: A prospective, observational, multicenter study was conducted over 56 weeks in adult patients with moderately to severely active UC who received ADA. Clinical response, remission, and mucosal healing were assessed using the Mayo score. Results: A total of 146 patients were enrolled from 17 academic hospitals. Clinical response rates were $52.1 \%$ and $37.7 \%$ and clinical remission rates were $24.0 \%$ and $22.0 \%$ at weeks 8 and 56 , respectively. Mucosal healing rates were $39.0 \%$ and $30.1 \%$ at weeks 8 and 56, respectively. Prior use of anti-tumor necrosis factor- $\alpha$ (anti-TNF- $\alpha$ ) did not affect clinical and endoscopic responses. The ADA drug level was significantly higher in patients with better outcomes at week $8(P<0.05)$. In patients with lower endoscopic activity, higher body mass index, and higher serum albumin levels at baseline, the clinical response rate was higher at week 8. In patients with lower Mayo scores and C-reactive protein levels, clinical responses, and mucosal healing at week 8 , the clinical response rate was higher at week 56 . Serious adverse drug reactions were identified in $2.8 \%$ of patients. Conclusions: ADA is effective and safe for induction and maintenance in Korean patients with UC, regardless of prior anti-TNF- $\alpha$ therapy. The ADA drug level is associated with the efficacy of induction therapy. Patients with better short-term outcomes were predictive of those with an improved long-term response. (Intest Res 2022;20:350-360)

Key Words: Inflammatory bowel disease; Tumor necrosis factor inhibitors; Treatment outcome 


\section{INTRODUCTION}

Ulcerative colitis (UC) is a chronic, idiopathic inflammatory bowel disease (IBD) characterized by relapsing abdominal pain and bloody diarrhea with or without mucus. ${ }^{1}$ The incidence and prevalence of UC have been reported as high in Northern Europe and North America and low in Asian countries. ${ }^{2-5}$ However, researchers recently observed an increased number of UC patients in Asian countries including Korea, Japan, China, and Taiwan. ${ }^{6-8}$ The incidence of UC in Asia has been rising in relation to rapid urbanization and a westernized lifestyle. ${ }^{9,10}$ A population-based study performed in Korea showed that the incidence and prevalence of UC have been gradually increasing. ${ }^{10,11}$ Furthermore, the genetic and clinical characteristics of IBD patients from Asia and Western countries are slightly different. ${ }^{12,13}$

Adalimumab (ADA) is a fully human immunoglobulin G1 monoclonal antibody directed against tumor necrosis factor- $\alpha$ (TNF- $\alpha$ ) that inhibits the activity of the cytokine by blocking the interaction of TNF- $\alpha$ with its p55 and p75 cell surface receptors. ${ }^{14}$ In Korea, ADA has been administered to patients with UC since 2013, and a nationwide population-based study showed that 27.6\% were treated using ADA among patients who received anti-TNF- $\alpha$ therapy. ${ }^{10}$

Till date, little information regarding the clinical outcomes of the use of ADA in the Korean population is available. Most published studies of clinical outcomes in IBD have been conducted on Western patients. Investigating the clinical outcomes of biologic agents in Korean IBD patients will enable a better understanding and optimal management of this condition. Therefore, we conducted a prospective, observational, multicenter study to evaluate the real-world efficacy and safety of ADA and predictors of response in Korean patients with UC.

\section{METHODS}

\section{Patients}

This prospective, observational, multicenter study was conducted at 17 academic hospitals in Korea from June 2015 to September 2018. The study protocol was approved by the institutional review board at each center (IRB No. C2015020) and registered at clinicaltrials.gov (study identifier: NCT02499263). All patients provided written informed consent. All work was carried out in compliance with the Ethical Principles for Medical Research Involving Human Subjects outlined in the Helsinki Declaration in 1975 (revised in 2000). All authors had access to the study data and reviewed and approved the final manuscript.

Eligible patients were aged $>18$ years and had moderately to severely active UC defined as a Mayo score ${ }^{15}$ between 6 to 12 , with an endoscopic subscore of at least 2, despite concurrent therapy with 5 -aminosalicylic acid, corticosteroids, and azathioprine/6-mercaptopurine. Previous use of anti-TNF- $\alpha$ agents other than ADA was permitted if the patient had discontinued its use owing to primary nonresponse, loss of response, or intolerance to the agent. Exclusion criteria were contraindications for using $\mathrm{ADA}$ including malignancy; severe infection such as active tuberculosis, invasive fungal infection, and opportunistic infection; being enrolled in other clinical trials; and pregnancy or breastfeeding. Patients were excluded if they chose to withdraw from the study or if the investigator discontinued ADA because of ethical or practical conflicts. Baseline assessment performed before administration of ADA, and details can be found in supplementary section.

\section{Study Design}

Patients received subcutaneous injections of ADA: $160 \mathrm{mg}$ at week $0,80 \mathrm{mg}$ at week 2 , and $40 \mathrm{mg}$ every alternate week from week 4. Patients were evaluated at weeks 0 (baseline), 8, 16, $24,32,40,48$, and 56 in accordance with clinical practice. The window period was permitted as \pm 1 week for every visit. All participants underwent safety evaluations $\leq 30$ days after the last administration of ADA.

Analysis of Mayo scores, including endoscopic subscore, and fecal calprotectin (FC) levels and laboratory tests, were performed at weeks 8 and 56. ADA trough level was evaluated at week 8 and at loss of response, even if ADA dose escalated to weekly injection. Patients with inadequate response to ADA (40 mg every 2 weeks) were permitted to escalate the dosage to $40 \mathrm{mg}$ every week. Patients with inadequate response to dose escalation discontinued the drug based on their physician's judgement. Inadequate response was defined in supplementary section.

\section{Outcomes}

The primary outcomes were clinical response rates at weeks 8 and 56. Clinical response was defined as a decrease in Mayo score from baseline by $\geq 3$ points and $\geq 30 \%$ with an accompanying decrease in rectal bleeding subscore of $\geq 1$ point or an absolute rectal bleeding subscore of 0 or 1 . Secondary outcomes were proportion of patients with clinical remission, steroid-free remission, and mucosal healing at weeks 8 and 56 . Clinical remission was defined as a Mayo score $\leq 2$ with no in- 
dividual subscore exceeding 1 point. Steroid-free remission was defined as a clinical remission status with no use of systemic corticosteroids for 12 weeks before the date of investigation. Mucosal healing was defined as an endoscopy subscore of 0 or 1 . Endoscopic remission was defined as an endoscopic subscore of 0 . Predictors of response at weeks 8 and 56 were evaluated based on clinical characteristics at baseline and week 8. Safety evaluation was performed according to the recent version of the Medical Dictionary for Regulatory Activities version 20.0. Physical examination, investigation of vital signs, and laboratory tests were performed from baseline to week 56 and after 30 days from the last administration.

\section{Statistical Methods}

Analyses were performed with the intent-to-treat set. Missing or incomplete data were handled using the nonresponder imputation method, i.e., patients with missing or incomplete data were assumed to have not achieved the endpoint. Continuous variables are described with the number of subjects, arithmetic mean, and standard deviation. For categorical variables, the frequency and proportion are presented. For all the statistical tests, two-sided tests were conducted at the 0.05 significance level using the independent or paired $t$-test and the chi-square test. To identify factors associated with clinical response at weeks 8 and 56, variables that were significant in univariate analysis were subsequently tested in multivariate logistic regression analysis and expressed as odds ratios (ORs) with 95\% confidence intervals (CIs). The Kaplan-Meier method was used to evaluate the drug persistence rate. All statistical analyses were performed using SAS version 9.4 (SAS Institute, Inc., Cary, NC, USA).

\section{RESULTS}

\section{Primary Endpoint}

A total of 146 patients from 17 academic hospitals in Korea were enrolled and included in the analysis. Table 1 summarizes the baseline clinical characteristics of the participants.

Clinical response was achieved in $52.1 \%(76 / 146)$ of the patients at week 8 and $37.7 \%(55 / 146)$ of the patients at week 56 (Fig. 1A). Clinical response rate at week 56 in week 8 responders was $54.0 \%$ (41/76). Clinical response rate was not significantly different between patients who received anti-TNF- $\alpha$ and those who did not (Fig. 1B and C). The combination therapy with azathioprine/6-mercaptopurine in baseline was not associated with clinical response rate at both weeks 8 and 56 (Fig. 1D).
Table 1. Baseline Demographic and Clinical Characteristics of Participants

\begin{tabular}{|c|c|}
\hline Characteristics & Participants $(n=146)$ \\
\hline Age (yr) & $44.9 \pm 14.9$ \\
\hline Male sex & $50(34.5)$ \\
\hline Body weight (kg) & $63.2 \pm 12.2$ \\
\hline BMI $\left(\mathrm{kg} / \mathrm{m}^{2}\right)^{\mathrm{a}}$ & $22.5 \pm 3.6$ \\
\hline Age at diagnosis (yr) & $39.4 \pm 15.5$ \\
\hline Duration of disease $(\mathrm{mo})^{b}$ & $52.8 \pm 49.6$ \\
\hline Mayo score & $8.7 \pm 1.4$ \\
\hline Endoscopic subscore & $2.5 \pm 0.5$ \\
\hline \multicolumn{2}{|l|}{ Disease location } \\
\hline Proctitis & $26(17.8)$ \\
\hline Left-sided colitis & $65(44.5)$ \\
\hline Extensive colitis & $50(34.3)$ \\
\hline Others & $5(3.4)$ \\
\hline \multicolumn{2}{|l|}{ Fecal calprotectin (mg/kg) } \\
\hline Mean $\pm S D$ & $894.6 \pm 630.4$ \\
\hline Median & 906.0 \\
\hline \multicolumn{2}{|l|}{ C-reactive protein (mg/dL) } \\
\hline Mean $\pm S D$ & $4.7 \pm 11.4$ \\
\hline Median & 0.9 \\
\hline \multicolumn{2}{|l|}{ Albumin (g/dL) } \\
\hline Mean $\pm S D$ & $3.8 \pm 0.6$ \\
\hline Median & 3.9 \\
\hline \multicolumn{2}{|l|}{ Concomitant medication (overlapped) } \\
\hline 5-Aminosalicylates & $133(94.3)$ \\
\hline Methotrexate & $3(2.1)$ \\
\hline Azathioprine/6-mercaptopurine & $79(56.0)$ \\
\hline Cyclosporine/tacrolimus & 0 \\
\hline Systemic corticosteroid & $59(41.8)$ \\
\hline 20 mg and above (daily dose) & $43(72.9)$ \\
\hline Less than 20 mg (daily dose) & $33(55.9)$ \\
\hline Prior anti-TNF therapy & $36(24.7)$ \\
\hline One medication & $34(94.4)$ \\
\hline Two medications and above & $2(5.6)$ \\
\hline
\end{tabular}

Values are presented as mean \pm standard deviation or number (\%).

aData on BMI were available for 144 patients.

${ }^{b}$ Data on duration of disease were available for 80 patients. $\mathrm{BMI}$, body mass index; TNF, tumor necrosis factor.

\section{Secondary Endpoints}

At week $8,24.0 \%(35 / 146)$ of patients were in clinical remission, and $22.0 \%$ (32/146) of patients achieved clinical remission at week 56. Steroid-free clinical remission was achieved in $12.3 \%(18 / 146)$ of patients at week 8 and $21.2 \%(31 / 146)$ of 

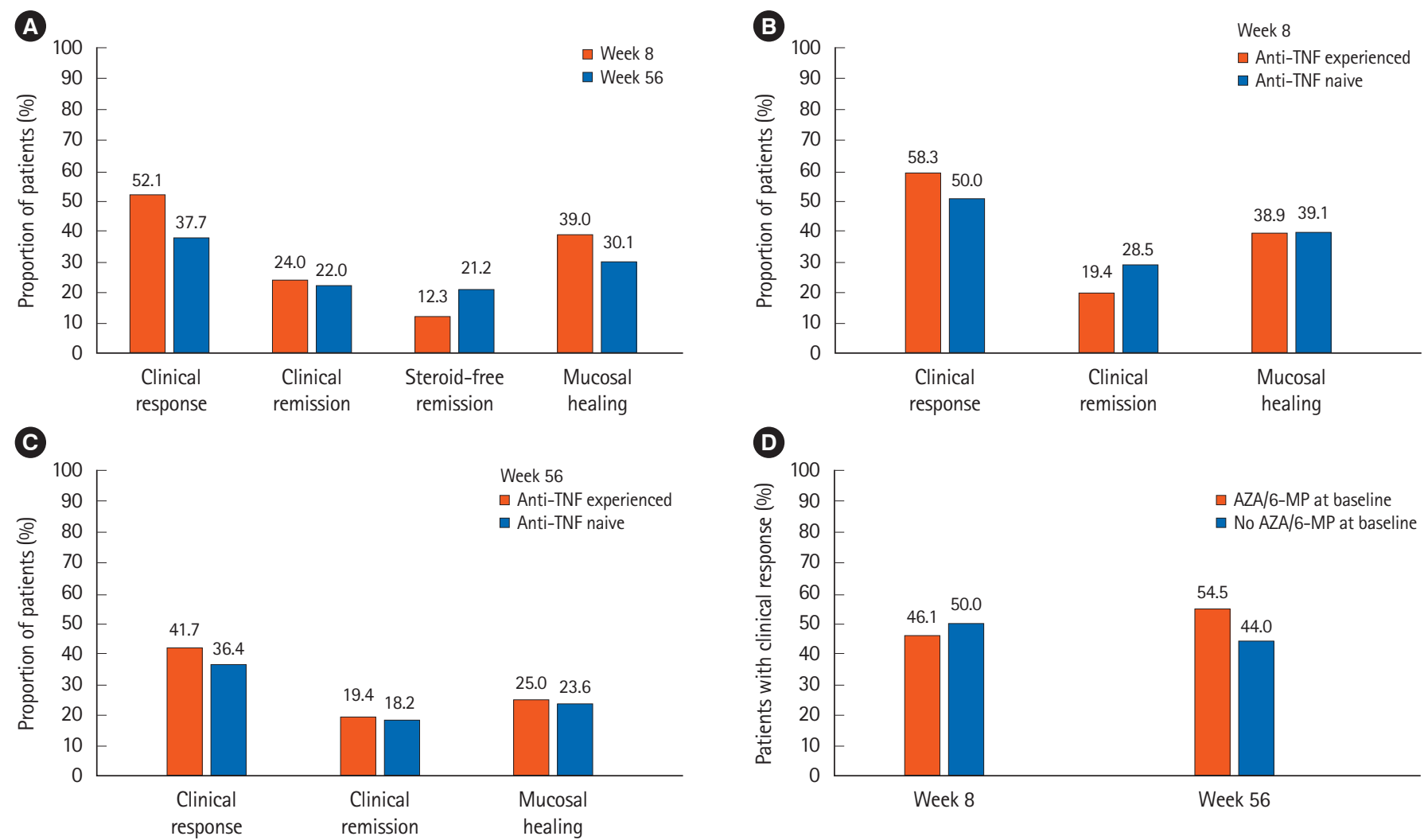

Fig. 1. Clinical response, remission, steroid-free remission, and mucosal healing rates according to the Mayo score at week 8 and 56 (A). Clinical outcomes according to prior anti-TNF- $\alpha$ therapy at week 8 (B) and at week 56 (C). Clinical response rates according to the use of immunomodulator at baseline (D). Clinical outcomes did not differ whether the patients had experienced anti-TNF therapy or not, and concomitant use of immunomodulator did not affect clinical response rate. TNF, tumor necrosis factor; AZA, azathioprine; 6-MP, 6-mercaptopurine.

patients at week 56. Mucosal healing was achieved in 39.0\% (57/146) of patients at week 8 and 30.1\% (44/146) at week 56 (Fig. 1A). Rates of clinical remission and mucosal healing were not significantly different between patients who received antiTNF- $\alpha$ and those who did not (Fig. 1B and C). Twenty-five patients (17.1\%) experienced dose escalation, and $40 \%$ and $20 \%$ of these achieved clinical response and remission at week 56 , respectively.

\section{Comparison of Clinical Characteristics between Clinical Responders and Nonresponders}

A comparison of clinical characteristics between clinical responders and nonresponders can be found in Tables 2 and 3 . Higher body mass index (BMI) $(P=0.034)$ and serum albumin level $(P=0.019)$, and less severe endoscopic findings $(P=0.002)$ at baseline were associated with clinical response at week 8 . Higher baseline BMI $(P=0.017)$, and clinical response $(P<0.001)$ and mucosal healing $(P=0.008)$ at week 8 were associated with clinical response at week 56 . Mayo score $(P=0.026)$ and serum C-reactive protein $(\mathrm{CRP})$ level $(P=0.035)$ at week 8 were significantly lower in clinical responders than in nonresponders at week 56. In the multivariate analysis, baseline non-severe endoscopic finding (OR, 2.951; 95\% CI, 1.365-6.382; $P=0.006)$ and clinical response (OR, 10.456; 95\% CI, 1.903-57.458; $P=$ $0.007)$ at week 8 were independent predictive factors for clinical responses at week 8 and week 56 , respectively (Table 4 ).

\section{Exploratory Outcomes}

Treatment persistence rate and causes of discontinuation can be found in Supplementary Fig. 1 and Supplementary Table 1. Clinical response rates according to the partial Mayo score can be found in Supplementary Fig. 2.

Mean serum ADA concentration (trough level, $\mu \mathrm{g} / \mathrm{mL}$ ) was significantly higher in patients who achieved clinical response (10.8 vs. $8.0, P=0.004$ ), remission (11.7 vs. $8.3, P=0.007$ ), and mucosal healing ( 11.0 vs. $8.5, P=0.010)$ than in those with no clinical response at week 8. Mean serum ADA level was 7.5 \pm 6.4 $\mu \mathrm{g} / \mathrm{mL}$ (range, $0.1-17.7 \mu \mathrm{g} / \mathrm{mL}$ ) in patients who stopped ADA 
Table 2. Comparison of Baseline Clinical Characteristics between Clinical Responders and Nonresponders at Week 8 Following Adalimumab Administration

\begin{tabular}{|c|c|c|c|}
\hline $\begin{array}{c}\text { Baseline clinical } \\
\text { characteristics }\end{array}$ & $\begin{array}{l}\text { Responder } \\
(n=76)\end{array}$ & $\begin{array}{c}\text { Non- } \\
\text { responder } \\
(n=70)\end{array}$ & $P$-value \\
\hline Age (yr) & $44.1 \pm 14.9$ & $45.9 \pm 15.0$ & 0.459 \\
\hline Male sex & $46(60.5)$ & $50(71.4)$ & 0.165 \\
\hline Body mass index $\left(\mathrm{kg} / \mathrm{m}^{2}\right)$ & $23.1 \pm 3.9$ & $21.8 \pm 3.2$ & 0.034 \\
\hline Mayo Clinic score & $8.7 \pm 1.4$ & $8.8 \pm 1.4$ & 0.582 \\
\hline Partial Mayo score & $6.2 \pm 1.2$ & $6.1 \pm 1.3$ & 0.558 \\
\hline Endoscopic finding & & & 0.002 \\
\hline Moderate & $44(57.9)$ & $23(32.9)$ & \\
\hline Severe & $32(42.1)$ & $47(67.1)$ & \\
\hline Disease location & & & 0.903 \\
\hline Proctitis & $14(18.4)$ & $12(17.1)$ & \\
\hline Left-sided colitis & $33(43.4)$ & $33(47.1)$ & \\
\hline Extensive colitis & $29(38.2)$ & $25(35.7)$ & \\
\hline Fecal calprotectin (mg/kg) & $853.7 \pm 620.9$ & $950.2 \pm 645.3$ & 0.414 \\
\hline C-reactive protein $(\mathrm{mg} / \mathrm{dL})$ & $3.3 \pm 6.7$ & $6.5 \pm 15.0$ & 0.109 \\
\hline Albumin $(\mathrm{g} / \mathrm{dL})$ & $3.9 \pm 0.6$ & $3.7 \pm 0.6$ & 0.019 \\
\hline \multicolumn{4}{|c|}{ Concomitant medication (overlap) } \\
\hline 5-Aminosalicylates & 68 (89.5) & $54(77.1)$ & 0.072 \\
\hline Azathioprine/6-MP & $35(46.1)$ & $35(50.0)$ & 0.633 \\
\hline Systemic corticosteroid & $25(32.9)$ & $19(27.1)$ & 0.449 \\
\hline
\end{tabular}

Values are presented as mean \pm standard deviation or number (\%). 6-MP, 6-mercaptopurine.

Table 4. Multivariate Analysis for Predictive Factors of Clinical Response to Adalimumab at Weeks 8 and 56

\begin{tabular}{lcc}
\hline Predictive factor & OR $(95 \% \mathrm{Cl})$ & $P$-value \\
\hline Clinical response at week 8 & & \\
Baseline & & \\
BMI $\geq 23 \mathrm{~kg} / \mathrm{m}^{2}$ & $1.218(0.578-2.567)$ & 0.604 \\
Non-severe endoscopic finding & $2.951(1.365-6.382)$ & 0.006 \\
Albumin $\geq 4.0 \mathrm{~g} / \mathrm{dL}$ & $1.497(0.697-3.214)$ & 0.301 \\
Clinical response at week 56 & & \\
Week 8 & & \\
Clinical response & $10.456(1.903-57.458)$ & 0.007 \\
Mucosal healing & $0.777(0.185-3.260)$ & 0.731 \\
Mayo score $\leq 3$ & $2.046(0.268-15.641)$ & 0.490 \\
Partial Mayo score $\leq 2$ & $0.296(0.040-2.189)$ & 0.233 \\
C-reactive protein $\leq 1 \mathrm{mg} / \mathrm{dL}$ & $1.748(0.479-6.375)$ & 0.398 \\
\hline
\end{tabular}

$\mathrm{OR}$, odds ratio; $\mathrm{Cl}$, confidence interval; $\mathrm{BMI}$, body mass index.
Table 3. Comparison of Clinical Characteristics between Clinical Responders and Nonresponders at Week 56 Following Adalimumab Administration

\begin{tabular}{|c|c|c|c|}
\hline Clinical characteristics & $\begin{array}{l}\text { Responder } \\
(n=55)\end{array}$ & $\begin{array}{l}\text { Non- } \\
\text { responder } \\
(n=91)\end{array}$ & $P$-value \\
\hline \multicolumn{4}{|l|}{ Baseline characteristics } \\
\hline Age (yr) & $43.8 \pm 14.9$ & $45.7 \pm 14.9$ & 0.460 \\
\hline Male sex & $33(60.0)$ & $63(69.2)$ & 0.255 \\
\hline Body mass index $\left(\mathrm{kg} / \mathrm{m}^{2}\right)$ & $23.4 \pm 3.9$ & $22.0 \pm 3.3$ & 0.017 \\
\hline Mayo Clinic score & $9.0 \pm 1.3$ & $8.6 \pm 1.4$ & 0.096 \\
\hline Partial Mayo score & $6.4 \pm 1.1$ & $6.0 \pm 1.3$ & 0.094 \\
\hline Endoscopic finding & & & 0.671 \\
\hline Moderate & $24(43.6)$ & $43(47.3)$ & \\
\hline Severe & $31(56.4)$ & $48(52.7)$ & \\
\hline Disease location & & & 0.304 \\
\hline Proctitis & 7 (12.7) & 19 (20.9) & \\
\hline Left-sided colitis & $24(43.6)$ & $42(46.2)$ & \\
\hline Extensive colitis & $24(43.6)$ & $30(33.0)$ & \\
\hline Fecal calprotectin (mg/kg) & $850.4 \pm 617.1$ & $920.8 \pm 640.9$ & 0.559 \\
\hline C-reactive protein (mg/dL) & $3.1 \pm 5.9$ & $5.9 \pm 15.0$ & 0.109 \\
\hline Albumin (g/dL) & $3.8 \pm 0.6$ & $3.8 \pm 0.6$ & 0.546 \\
\hline \multicolumn{4}{|c|}{ Concomitant medication (overlap) } \\
\hline 5-Aminosalicylates & 49 (89.1) & $73(80.2)$ & 0.161 \\
\hline Azathioprine/6-MP & $30(54.5)$ & $40(44.0)$ & 0.215 \\
\hline Systemic corticosteroid & $17(30.9)$ & $27(29.7)$ & 0.874 \\
\hline \multicolumn{4}{|l|}{ Characteristics at week 8} \\
\hline Clinical response & $41(74.5)$ & $35(38.5)$ & $<0.001$ \\
\hline Mucosal healing & $29(52.7)$ & $28(30.8)$ & 0.008 \\
\hline Mayo score & $3.2 \pm 2.3$ & $4.4 \pm 3.1$ & 0.026 \\
\hline Partial Mayo score & $1.9 \pm 1.6$ & $3.0 \pm 2.3$ & 0.001 \\
\hline C-reactive protein (mg/dL) & $0.9 \pm 1.3$ & $2.2 \pm 4.9$ & 0.035 \\
\hline Fecal calprotectin (mg/kg) & $422.5 \pm 505.7$ & $317.7 \pm 435.8$ & 0.187 \\
\hline Albumin (g/dL) & $4.2 \pm 0.5$ & $4.0 \pm 0.5$ & 0.139 \\
\hline $\begin{array}{l}\text { Adalimumab trough level } \\
(\mu \mathrm{g} / \mathrm{mL})\end{array}$ & $9.3 \pm 5.0$ & $9.9 \pm 5.4$ & 0.550 \\
\hline $\begin{array}{l}\text { Concomitant use of } \\
\text { azathioprine/6-MP }\end{array}$ & $27(49.1)$ & $31(34.1)$ & 0.072 \\
\hline
\end{tabular}

Values are presented as mean \pm standard deviation or number (\%). 6-MP, 6-mercaptopurine.

administration because of inadequate response (Fig. 2). FC and CRP levels associated with the clinical and endoscopic outcomes can be found in Fig. 3 .

A total of 246 FC levels were collected at the 3 points, and the correlation with endoscopic findings was investigated by 

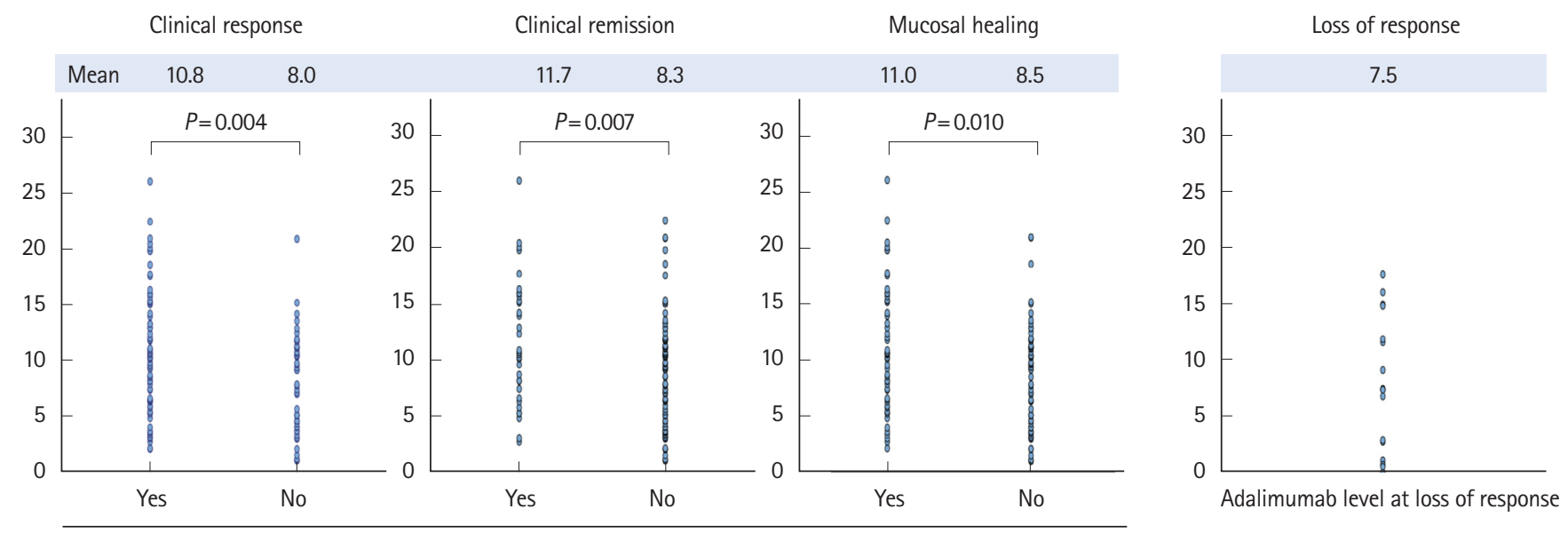

Adalimumab level at week 8

Fig. 2. Serum adalimumab concentration $(\mu \mathrm{g} / \mathrm{mL})$ according to clinical outcomes at week 8 . Mean serum adalimumab levels were significantly higher in patients who achieved clinical response, remission, and mucosal healing than in those without clinical response, remission, and mucosal healing, respectively, at week 8.
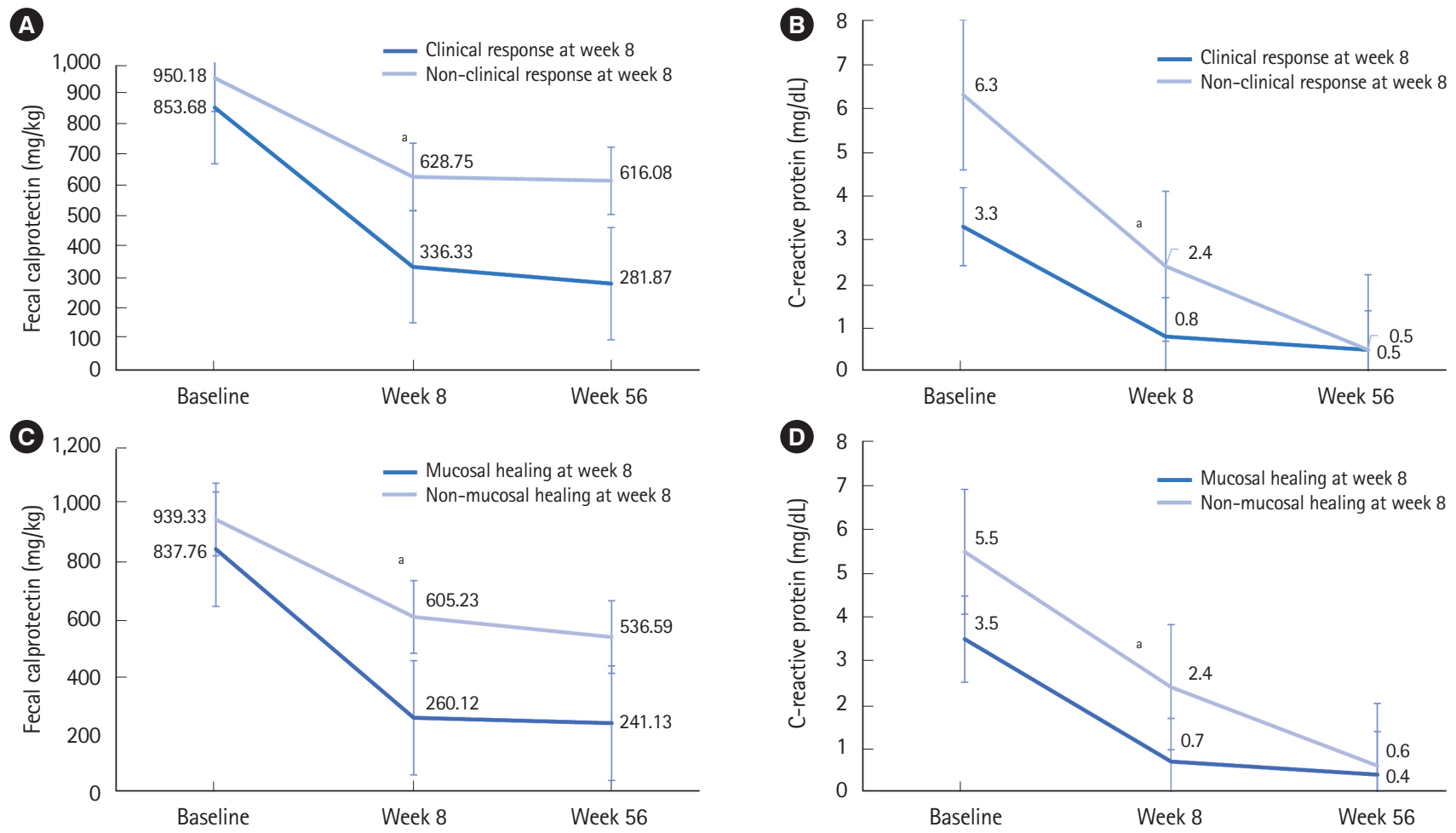

Fig. 3. Changes in mean fecal calprotectin and C-reactive protein levels from baseline in clinical responders and nonresponders at week 8 $(A, B)$ and in patients with and without mucosal healing at week $8(C, D)$. Fecal calprotectin and C-reactive protein levels significantly improved in patients achieving clinical response and mucosal healing compared with patients without clinical response and mucosal healing, respectively, at week $8 .{ }^{a} P<0.05$.

integrating them (Fig. 4). The FC level to predict mucosal healing was $274.7 \mathrm{mg} / \mathrm{kg}$, with a sensitivity of $72.2 \%$ and specificity of $71.3 \%$ on the receiver operating curve (area under the curve, 0.771 ). The predictive level for endoscopic remission
(Mayo subscore 0) was $87.9 \mathrm{mg} / \mathrm{kg}$, with a sensitivity of $73.6 \%$ and specificity of $73.5 \%$ on the receiver operating curve (area under the curve, 0.774). 
A

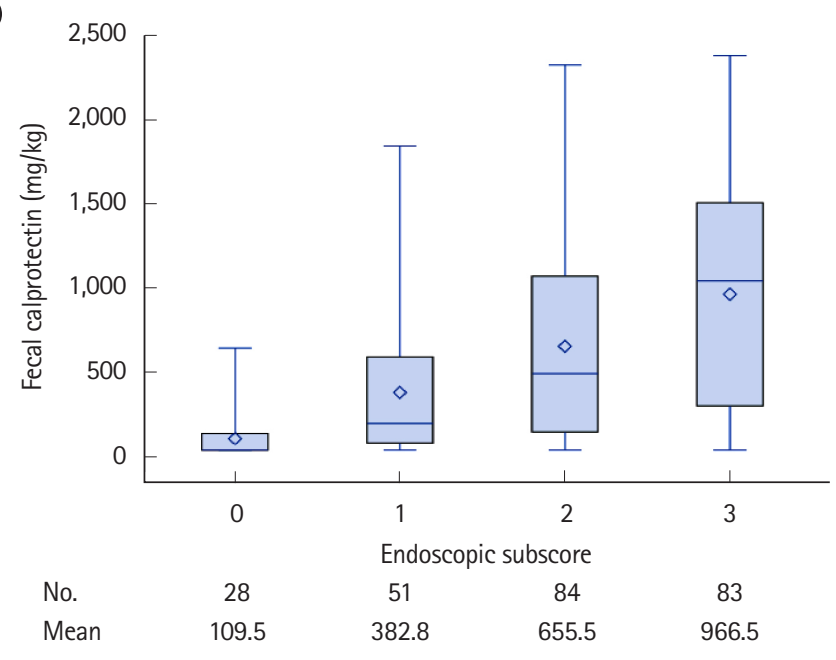

$\boldsymbol{0}$

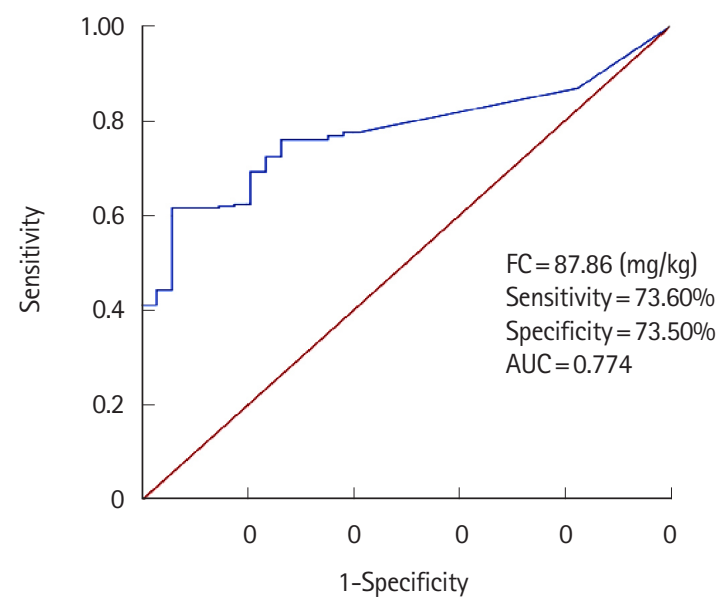

\section{Safety}

During the study period, $6.2 \%$ (9/146) of patients experienced adverse drug reactions. Serious adverse drug reactions that induced hospital admission were identified in 2.8\% (4/146) of patients as follows: pneumonia, pulmonary tuberculosis, renal failure, and abdominal pain (Supplementary Table 2).

\section{DISCUSSION}

In this prospective multicenter study, we evaluated the realworld clinical effectiveness of ADA treatment and factors associated with the clinical response in Korean patients with moderately to severely active UC. Our study showed similar or slightly higher rates of clinical response and remission than 2 previous pivotal studies conducted in Western countries: ULTRA- 1 and ULTRA-2 (response rates, 50.4\%-54.6\%; remission rates, $16.5 \%-18.5 \%$ at week 8 and $30.2 \%-17.3 \%$ at week 52). ${ }^{14,16}$ Several studies investigating the real-world efficacy of ADA have been reported worldwide. Although it is difficult to

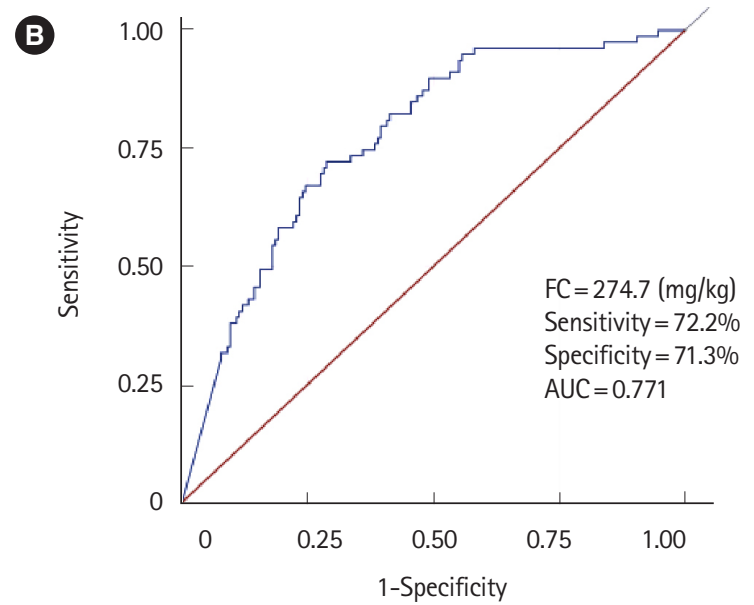

Fig. 4. Fecal calprotectin (FC) levels according to endoscopic subscore (A), FC level to predict mucosal healing (B) and endoscopic remission (C) on receiver operating characteristic (ROC) curve. FC levels were well correlated with patients' endoscopic activities. The predictive level was $274.8 \mathrm{mg} / \mathrm{kg}$ for mucosal healing and $98 \mathrm{mg} / \mathrm{kg}$ for endoscopic remission, respectively. $A U C$, area under the ROC curve.

directly compare these results, because each study defined clinical response and remission differently, they show similar trends in outcomes. ${ }^{17-19}$ A Japanese real-world study that applied the same definition for clinical response and remission as this study, reported similar outcomes. ${ }^{17}$ The mucosal healing rate in our study tended to be lower than that reported in previous Western studies, ${ }^{18,19}$ but similar to that reported in a Japanese study. ${ }^{17}$ Collectively, ADA was similarly effective for induction and maintenance treatments in Korean patients with active UC who were unresponsive to corticosteroids and/ or azathioprine/6-mercaptopurine.

Previous experience with anti-TNF- $\alpha$ therapy was not found to impact the short- or long-term outcomes in our study. Prior anti-TNF- $\alpha$ therapy has been reported to have controversial effects on clinical outcomes of ADA in patients with UC. The ULTRA-2 and ULTRA-3 studies reported better outcomes in patients not treated with anti-TNF- $\alpha .{ }^{14,20}$ A retrospective multicenter study in Spain investigated the influence of previous anti-TNF- $\alpha$ use on the outcomes of ADA maintenance treat- 
ment in patients with UC; patients not previously treated with anti-TNF- $\alpha$ had a numerically higher rate of clinical response at week 56 without statistical significance. ${ }^{21}$ They had significantly lower probabilities of avoiding colectomy and dose escalation. However, in a previous Hungarian prospective study, response to ADA and need for dose escalation were not associated with previous infliximab (IFX) therapy. ${ }^{19}$ A retrospective study performed in Ireland showed a trend towards better outcomes in patients previously received anti-TNF- $\alpha$ compared with those who were not. ${ }^{22}$ It is difficult to explain this inconsistency. Relatively small portion of patients with antiTNF- $\alpha(n=36,24.7 \%)$, and higher rate of dose escalation in patient with anti-TNF exposure (41.9\%) compared with anti-TNF naïve patients (9.0\%) (data not shown) in our study may affect the results. Although a note of caution is due, ADA can be suggested as a beneficial option for Korean patients with moderately to severely active UC treated previously with anti-TNF- $\alpha$ therapy. In addition, the combination therapy with azathioprine/ 6-mercaptopurine did not affect clinical response rate. This finding is consistent with that of previous studies showing no efficacy-related benefits following immunomodulator/ADA combination therapy. ${ }^{20,23,24}$

In this study, baseline BMI, endoscopic findings, and serum albumin level were associated with clinical response at week 8. At week 56, baseline BMI and clinical response, mucosal healing, CRP level, and Mayo score at week 8 were associated with clinical response. In the multivariate analysis, baseline non-severe endoscopic finding and clinical response at week 8 were independent factors for predicting response at weeks 8 and 52, respectively. Although it was a significant factor only in the univariate analysis, BMI was associated with both shortand long-term response. Previous studies have shown that obese patients tended to have higher risk of nonresponse to biologic agents because of their direct effect on inflammation and modification of pharmacokinetics. ${ }^{14,25,26}$ However, average BMI of both responder and nonresponder groups were within the normal range in our study. Relatively higher BMI within the normal range might reflect less severe disease status. CRP level has been suggested as a predictor of poor outcome in UC patients ${ }^{27}$ and considered a biomarker of response to IFX induction therapy. ${ }^{28,29}$ Endoscopic finding is also one of the major factors determining the severity and prognosis of UC. In this study, the baseline CRP levels were lower in responders than in nonresponders, though without statistical significance. The less severe endoscopic activity was associated with better response to induction therapy of ADA. The findings from this study suggest that ADA therapy may be more effective in moderately active UC than in severely active UC.

Parameters associated with early response such as mucosal healing, clinical response, and CRP level were associated with long-term response. Mucosal healing has been reported to be associated with long-term clinical outcomes ${ }^{18,30}$ and suggested as a predictive factor of long-term outcome in Korean UC patients treated with IFX. ${ }^{31}$ Early clinical response has also been demonstrated as a predictive factor of better long-term clinical outcomes in several real-world studies. ${ }^{17,32,33}$ In Korean patients with moderately to severely active UC, early response is also a positive predictor for long-term clinical response.

During the study period, 25 patients (17.1\%) required dose escalation, and $40 \%$ and $20 \%$ of these regained clinical response and remission, respectively, at week 56. Compared to previous Western studies, the proportion of patients who experienced dose escalation in our study was relatively small; however, the clinical outcomes are similar to those of these studies. $^{21,34,35}$

Consistent with previous studies on mucosal healing-associated FC levels, ${ }^{36-38}$ in this study, FC levels were well correlated with not only patients' clinical outcomes, but also endoscopic activities. The predictive level was $274.8 \mathrm{mg} / \mathrm{kg}$ for mucosal healing and $98 \mathrm{mg} / \mathrm{kg}$ for endoscopic remission. These novel findings can be used to predict endoscopic activities in UC patients.

Serum ADA concentrations (trough level) at week 8 were associated significantly with clinical outcomes of induction therapy. The mean ADA concentrations in patients without clinical response, remission, and mucosal healing were relatively higher compared with those in previous studies. ${ }^{39-41}$ It can be assumed that different mechanisms other than signaling pathway via TNF may be involved in the development of UC in these patients. In addition, most previous studies included both patients with UC and Crohn's disease, and few studies have been conducted to investigate ADA concentration in patients with UC only. A Belgian study including IFX responders and nonresponders showed similar ADA concentrations as those in this study with respect to short-term mucosal healing. $^{42}$ The researchers reported that the average ADA concentration of patients with mucosal healing at week 4 was $10.6 \mu \mathrm{g} / \mathrm{mL}$, which was significantly higher than the concentration in those without mucosal healing $(7.4 \mu \mathrm{g} / \mathrm{mL}, P=0.014)$. More real-world data may be needed to establish optimal level for treatment target, but our finding suggests that the higher the drug concentration at week 8 after $\mathrm{ADA}$ induction therapy, 
the better was the expected clinical effect.

No new safety signals were observed in the present study, and the incidence rate was similar to that described in other studies. ${ }^{18,43}$ Any different tendency in safety from the approved label of ADA was not observed. Patients with severe adverse drug reactions including abdominal pain and pulmonary tuberculosis were treated properly, and no deaths were reported.

This was an observational study in routine clinical practice, having certain inherent limitations such as the lack of randomization, leading to potential bias. Moreover, the proportion of subjects who completed the evaluation without any major protocol deviation among the intent-to-treat set was relatively small. Therefore, with a small sample size, these data must be interpreted with caution. Furthermore, anti-ADA antibodies were not evaluated in this study, although it is used in clinical practice in Western countries. However, this study was the first multicenter prospective study to evaluate the efficacy and safety of ADA in Korean UC patients in the real-life clinical setting and explore clinical predictors of response to $\mathrm{ADA}$, including FC and ADA drug levels.

In conclusion, this study showed that ADA is effective and safe for Korean patients with moderately to severely active UC regardless of prior anti-TNF- $\alpha$ therapy. ADA drug level is associated with the efficacy of induction therapy. A good response to induction therapy suggests positive long-term outcomes in Korean patients with moderately to severely active UC.

\section{ADDITIONAL INFORMATION}

\section{Funding Source}

This work was funded by AbbVie Ltd., North Chicago, IL, USA and the National Research Foundation of Korea (NRF) (Grant/Award No. NRF-2020R1F1A1075489). AbbVie was responsible for the study design, interpretation of data, reviewing and approving of the publication.

\section{Conflict of Interest}

There are no conflicts of interest to disclose, except for Teng D: employees of AbbVie, Inc. and may hold stock or stock options. Kim DH is a former employee of AbbVie, Inc.

Kim JW, Park DI, Kim YS are editorial board members of the journal but were not involved in the peer reviewer selection, evaluation, or decision process of this article. No other potential conflicts of interest relevant to this article were reported.

\section{Data Availability Statement}

Not applicable.

\section{Author Contribution}

Designed the study: Choi CH, Park SJ, Im JP, Kim HJ, Jung SA, Kim HK, Kim JW, Lee KM, Lee J, Kang SB, Shin SJ, Kim YS, Kim TO, Kim HS, Park DI, Kim Eun Sun, Kim Eun Soo, Kim YH, Kim DH, Teng D. Data curation: Shin SY, Kim Y, Choi CH. Formal analysis: Shin SY, Kim Y, Kim JH, Kim W. Visualization: Shin SY, Kim Y, Choi CH. Writing - original draft: Shin SY, Park SJ. Writing - review \& editing: Choi CH, Im JP, Kim HJ, Jung SA, Kim JW, Lee KM, Lee J, Kang SB, Shin SJ, Kim YS, Kim TO, Kim HS, Park DI, Kim HK, Kim Eun Sun, Kim Eun Soo, Kim YH. Approval of final manuscript: all authors.

\section{ORCID}

Shin SY

Park SJ

Kim Y

$\operatorname{Im} J P$

Kim HJ

Lee KM

Kim JW

Jung SA

Lee J

Kang SB

Shin SJ

Kim ES

Kim YS

Kim TO

Kim HS

Park DI

Kim HK

Kim ES

Kim YH

Kim DH

Teng D

Kim JH

Kim W

Choi CH

https://orcid.org/0000-0001-8970-2444 https://orcid.org/0000-0003-2963-1394 https://orcid.org/0000-0003-0682-4046 https://orcid.org/0000-0003-1584-0160 https://orcid.org/0000-0002-9675-4557 https://orcid.org/0000-0003-2850-4553 https://orcid.org/0000-0002-1214-5544 https://orcid.org/0000-0001-7224-2867 https://orcid.org/0000-0002-8060-9646 https://orcid.org/0000-0002-1946-7896 https://orcid.org/0000-0003-1849-4435 https://orcid.org/0000-0002-5235-4332 https://orcid.org/0000-0002-5156-3458 https://orcid.org/0000-0002-6369-5618 https://orcid.org/0000-0003-4834-0496 https://orcid.org/0000-0003-2307-8575 https://orcid.org/0000-0003-4230-3897 https://orcid.org/0000-0003-0806-9136 https://orcid.org/0000-0003-1803-2513 https://orcid.org/0000-0003-0064-1732 https://orcid.org/0000-0001-5520-8915 https://orcid.org/0000-0002-3343-4820 https://orcid.org/0000-0001-9649-3919 https://orcid.org/0000-0001-7089-532X

\section{Supplementary Material}

Supplementary materials are available at the Intestinal Research website (https://www.irjournal.org). 


\section{REFERENCES}

1. Danese S, Fiocchi C. Ulcerative colitis. N Engl J Med 2011;365: 1713-1725.

2. Cosnes J, Gower-Rousseau C, Seksik P, Cortot A. Epidemiology and natural history of inflammatory bowel diseases. Gastroenterology 2011;140:1785-1794.

3. Molodecky NA, Soon IS, Rabi DM, et al. Increasing incidence and prevalence of the inflammatory bowel diseases with time, based on systematic review. Gastroenterology 2012;142:4654.

4. Loftus CG, Loftus EV Jr, Harmsen WS, et al. Update on the incidence and prevalence of Crohn's disease and ulcerative colitis in Olmsted County, Minnesota, 1940-2000. Inflamm Bowel Dis 2007;13:254-261.

5. Yang SK, Loftus EV Jr, Sandborn WJ. Epidemiology of inflammatory bowel disease in Asia. Inflamm Bowel Dis 2001;7:260270.

6. Lakatos PL. Recent trends in the epidemiology of inflammatory bowel diseases: up or down? World J Gastroenterol 2006; 12:6102-6108.

7. Ng WK, Wong SH, Ng SC. Changing epidemiological trends of inflammatory bowel disease in Asia. Intest Res 2016;14:111119.

8. Yen HH, Weng MT, Tung CC, et al. Epidemiological trend in inflammatory bowel disease in Taiwan from 2001 to 2015: a nationwide populationbased study. Intest Res 2019;17:54-62.

9. Prideaux L, Kamm MA, De Cruz PP, Chan FK, Ng SC. Inflammatory bowel disease in Asia: a systematic review. J Gastroenterol Hepatol 2012;27:1266-1280.

10. Jung YS, Han M, Kim WH, Park S, Cheon JH. Incidence and clinical outcomes of inflammatory bowel disease in South Korea, 2011-2014: a nationwide population-based study. Dig Dis Sci 2017;62:2102-2112.

11. Park SH, Kim YJ, Rhee KH, et al. A 30-year trend analysis in the epidemiology of inflammatory bowel disease in the Songpa-Kangdong district of Seoul, Korea in 1986-2015. J Crohns Colitis 2019;13:1410-1417.

12. Lee JH, Cheon JH, Kim ES, et al. The prevalence and clinical significance of perinuclear anti-neutrophil cytoplasmic antibody in Korean patients with ulcerative colitis. Dig Dis Sci 2010;55:1406-1412.

13. Park SH, Kim YM, Yang SK, et al. Clinical features and natural history of ulcerative colitis in Korea. Inflamm Bowel Dis 2007; 13:278-283

14. Sandborn WJ, van Assche G, Reinisch W, et al. Adalimumab induces and maintains clinical remission in patients with moderate-to-severe ulcerative colitis. Gastroenterology 2012;142: 257-265.

15. Schroeder KW, Tremaine WJ, Ilstrup DM. Coated oral 5-aminosalicylic acid therapy for mildly to moderately active ulcerative colitis: a randomized study. N Engl J Med 1987;317:16251629.

16. Reinisch W, Sandborn WJ, Hommes DW, et al. Adalimumab for induction of clinical remission in moderately to severely active ulcerative colitis: results of a randomised controlled trial. Gut 2011;60:780-787.

17. Suzuki Y, Motoya S, Hanai H, et al. Efficacy and safety of adalimumab in Japanese patients with moderately to severely active ulcerative colitis. J Gastroenterol 2014;49:283-294.

18. Muñoz-Villafranca C, Ortiz de Zarate J, Arreba P, et al. Adalimumab treatment of anti-TNF-naïve patients with ulcerative colitis: deep remission and response factors. Dig Liver Dis 2018; 50:812-819.

19. Bálint A, Farkas K, Palatka K, et al. Efficacy and safety of adalimumab in ulcerative colitis refractory to conventional therapy in routine clinical practice. J Crohns Colitis 2016;10:26-30.

20. Dulai PS, Siegel CA, Colombel JF, Sandborn WJ, Peyrin-Biroulet L. Systematic review: monotherapy with antitumour necrosis factor $\alpha$ agents versus combination therapy with an immunosuppressive for IBD. Gut 2014;63:1843-1853.

21. Taxonera C, Iglesias E, Muñoz F, et al. Adalimumab maintenance treatment in ulcerative colitis: outcomes by prior antiTNF use and efficacy of dose escalation. Dig Dis Sci 2017;62: 481-490.

22. Hussey M, Mc Garrigle R, Kennedy U, et al. Long-term assessment of clinical response to adalimumab therapy in refractory ulcerative colitis. Eur J Gastroenterol Hepatol 2016;28:217221.

23. Colombel JF, Jharap B, Sandborn WJ, et al. Effects of concomitant immunomodulators on the pharmacokinetics, efficacy and safety of adalimumab in patients with Crohn's disease or ulcerative colitis who had failed conventional therapy. Aliment Pharmacol Ther 2017;45:50-62.

24. Matsumoto T, Motoya S, Watanabe K, et al. Adalimumab monotherapy and a combination with azathioprine for Crohn's disease: a prospective, randomized trial. J Crohns Colitis 2016; 10:1259-1266.

25. Harper JW, Sinanan MN, Zisman TL. Increased body mass index is associated with earlier time to loss of response to infliximab in patients with inflammatory bowel disease. Inflamm Bowel Dis 2013;19:2118-2124. 
26. Dahiya DS, Kichloo A, Wani F, Singh J, Solanki D, Shaka H. A nationwide analysis on the influence of obesity in inflammatory bowel disease hospitalizations. Intest Res 2022;20:342349.

27. Henriksen M, Jahnsen J, Lygren I, et al. C-reactive protein: a predictive factor and marker of inflammation in inflammatory bowel disease: results from a prospective population-based study. Gut 2008;57:1518-1523.

28. Iwasa R, Yamada A, Sono K, Furukawa R, Takeuchi K, Suzuki Y. C-reactive protein level at 2 weeks following initiation of infliximab induction therapy predicts outcomes in patients with ulcerative colitis: a 3 year follow-up study. BMC Gastroenterol 2015;15:103.

29. Con D, Andrew B, Nicolaides S, van Langenberg DR, Vasudevan A. Biomarker dynamics during infliximab salvage for acute severe ulcerative colitis: C-reactive protein (CRP)-lymphocyte ratio and CRP-albumin ratio are useful in predicting colectomy. Intest Res 2022;20:101-113.

30. Ardizzone S, Cassinotti A, Duca P, et al. Mucosal healing predicts late outcomes after the first course of corticosteroids for newly diagnosed ulcerative colitis. Clin Gastroenterol Hepatol 2011;9:483-489.

31. Lee KM, Jeen YT, Cho JY, et al. Efficacy, safety, and predictors of response to infliximab therapy for ulcerative colitis: a Korean multicenter retrospective study. J Gastroenterol Hepatol 2013;28:1829-1833.

32. Italian Group for the Study of Inflammatory Bowel Disease, Armuzzi A, Biancone L, et al. Adalimumab in active ulcerative colitis: a "real-life" observational study. Dig Liver Dis 2013;45: 738-743.

33. Taxonera C, Estellés J, Fernández-Blanco I, et al. Adalimumab induction and maintenance therapy for patients with ulcerative colitis previously treated with infliximab. Aliment Pharmacol Ther 2011;33:340-348.

34. Van de Vondel S, Baert F, Reenaers C, et al. Incidence and predictors of success of adalimumab dose escalation and de-escalation in ulcerative colitis: a real-world Belgian cohort study.
Inflamm Bowel Dis 2018;24:1099-1105.

35. Wolf D, D'Haens G, Sandborn WJ, et al. Escalation to weekly dosing recaptures response in adalimumab-treated patients with moderately to severely active ulcerative colitis. Aliment Pharmacol Ther 2014;40:486-497.

36. Lin WC, Wong JM, Tung CC, et al. Fecal calprotectin correlated with endoscopic remission for Asian inflammatory bowel disease patients. World J Gastroenterol 2015;21:13566-13573.

37. Lee SH, Kim MJ, Chang K, et al. Fecal calprotectin predicts complete mucosal healing and better correlates with the ulcerative colitis endoscopic index of severity than with the Mayo endoscopic subscore in patients with ulcerative colitis. BMC Gastroenterol 2017;17:110.

38. Kristensen V, Røseth A, Ahmad T, Skar V, Moum B. Fecal calprotectin: a reliable predictor of mucosal healing after treatment for active ulcerative colitis. Gastroenterol Res Pract 2017; 2017:2098293.

39. Yarur AJ, Jain A, Hauenstein SI, et al. Higher adalimumab levels are associated with histologic and endoscopic remission in patients with Crohn's disease and ulcerative colitis. Inflamm Bowel Dis 2016;22:409-415.

40. Bodini G, Giannini EG, Savarino V, et al. Adalimumab trough serum levels and anti-adalimumab antibodies in the longterm clinical outcome of patients with Crohn's disease. Scand J Gastroenterol 2016;51:1081-1086.

41. Paul S, Moreau AC, Del Tedesco E, et al. Pharmacokinetics of adalimumab in inflammatory bowel diseases: a systematic review and meta-analysis. Inflamm Bowel Dis 2014;20:12881295.

42. Papamichael K, Baert F, Tops S, et al. Post-induction adalimumab concentration is associated with short-term mucosal healing in patients with ulcerative colitis. J Crohns Colitis 2017; 11:53-59.

43. Ogata H, Hagiwara T, Kawaberi T, Kobayashi M, Hibi T. Safety and effectiveness of adalimumab in the treatment of ulcerative colitis: results from a large-scale, prospective, multicenter, observational study. Intest Res 2021;19:419-429. 
See "Clinical outcomes and predictors of response for adalimumab in patients with moderately to severely active ulcerative colitis: a KASID prospective multicenter cohort study" on pages 350-360.

Supplementary Table 1. Patients Who Discontinued Adalimumab and Related Reasons

\begin{tabular}{lc}
\hline Related reason & Value $(\mathrm{n}=62)$ \\
\hline Lack of efficacy & $34(54.8)$ \\
Adverse event & $11(17.7)$ \\
Follow-up loss & $9(14.5)$ \\
Subject's decision & $6(9.7)$ \\
Pregnancy & $1(1.6)$ \\
Others & $1(1.6)$ \\
\hline
\end{tabular}

Values are presented as number (\%). 
Supplementary Table 2. Adverse Drug Reactions with Adalimumab

\begin{tabular}{ll}
\hline Variable & $\begin{array}{c}\text { Value } \\
(\mathrm{n}=146)\end{array}$ \\
\hline Adverse drug reaction & \\
Gastrointestinal disorders & $3(2.1)$ \\
Hematochezia & $2(1.4)$ \\
Abdominal pain & $1(0.7)$ \\
Infections and infestations & $3(2.1)$ \\
Cytomegalovirus infection & $1(0.7)$ \\
Pneumonia & $1(0.7)$ \\
Pulmonary tuberculosis & $1(0.7)$ \\
General disorders and administration site conditions & $1(0.7)$ \\
Injection site reaction & $1(0.7)$ \\
Renal and urinary disorders & $1(0.7)$ \\
Renal failure & $1(0.7)$ \\
Skin and subcutaneous tissue disorders & $1(0.7)$ \\
Swelling face & $1(0.7)$ \\
Total & $9(6.2)$ \\
Severe adverse drug reaction & $1(0.7)$ \\
Gastrointestinal disorders & $1(0.7)$ \\
Abdominal pain & $1(0.7)$ \\
Infections and infestations & $1(0.7)$ \\
Pneumonia & $4(2.8)$ \\
Rulmonary tuberculosis & $1(0.7)$ \\
\hline
\end{tabular}

Values are presented as number (\%).

Adverse drug reaction: adverse events whose casualty with the adalimumab is "Probable," "Possible," "Probably not," "Not assessable." 


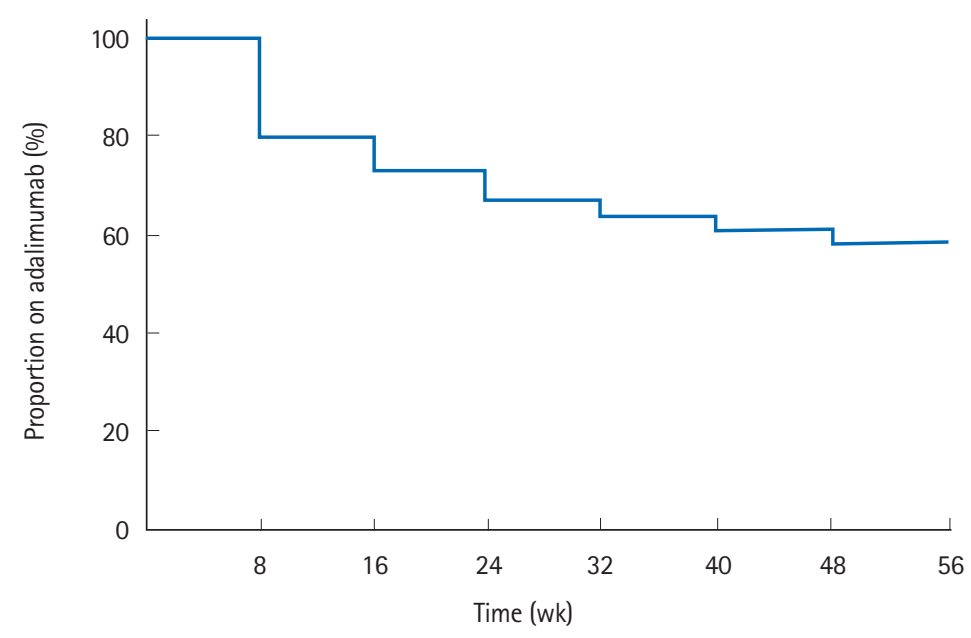

No. of patients

146

129

114

105

96

92

88

84

Supplementary Fig. 1. Proportion of patients remaining on adalimumab during the follow-up period. 


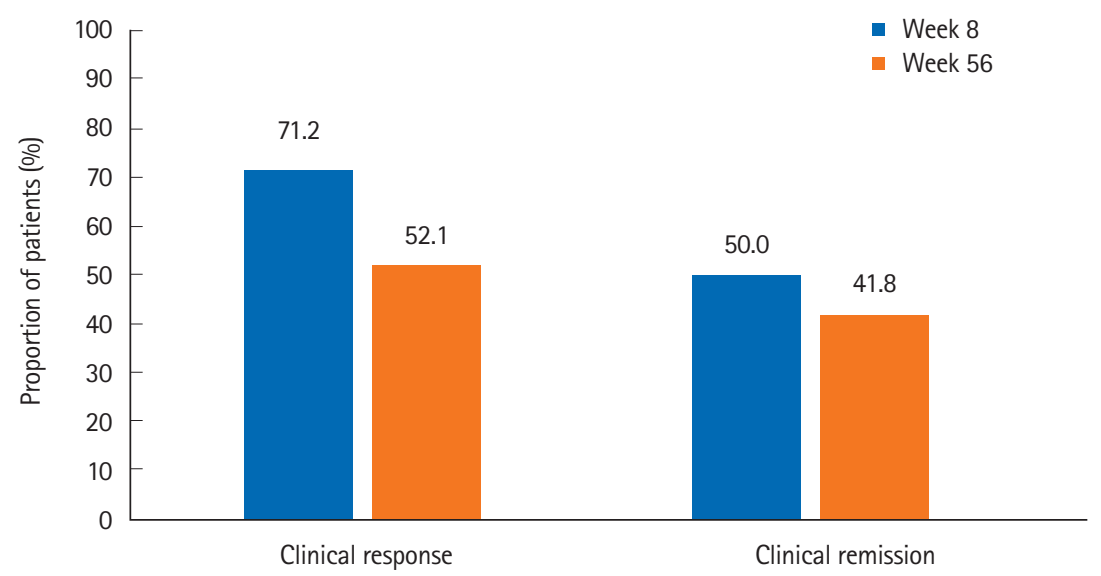

Supplementary Fig. 2. Proportion of patients with clinical response and remission according to partial Mayo Clinic score. 